\title{
Modeling and Analyzing Engagement in Social Network Challenges
}

\author{
Marco Brambilla, Stefano Ceri, Chiara Leonardi, \\ Andrea Mauri, Riccardo Volonterio \\ Dipartimento di Elettronica, Informazione e Bioingegneria (DEIB) \\ Politecnico di Milano. Piazza Leonardo da Vinci, 32. 20133 Milano, Italy \\ Email: $\{$ name.surname\}@polimi.it
}

\begin{abstract}
Participation to challenges within social networks is a very effective instrument for promoting a brand or event. In this paper, we take the challenge organizer's perspective, and we study how to raise the engagement of players in challenges where the players are stimulated to create and evaluate content, thereby indirectly raising the awareness about the brand or event itself. We illustrate a comprehensive model of the actions and strategies that can be exploited for progressively boosting the social engagement during the challenge evolution. The model studies the organizer-driven management of interactions among players, and evaluates the effectiveness of each action in light of several other factors (time, repetition, third party actions, interplay between different social networks, and so on). We evaluate the model through a set of experiment upon a real case, the YourExpo2015 challenge. Overall, our experiments lasted 9 weeks and mobilized hundreds of thousands of users on two different social platforms; our quantitative analysis assesses the validity of the model.
\end{abstract}

\section{Introduction}

Social networks are an essential aspect of communication strategies for promoting events and brands; effectiveness of communication is typically measured in terms of the intensity and quality of engagement of its members. This paper is concerned with the modeling and analysis of social challenges whose main purpose is to collect content produced by users in connection to a specific brand or event.

In this context, the objective of this paper is to define a model for social challenges, which allows challenge managers to define the appropriate strategies for increasing both participation and content creation, using multiple social networks, over a planned lifetime, in the presence of players, fans, and owners. More precisely, the research questions we want to investigate are the following:

1. Can we identify different roles in an online challenge, based on their activity?

2. Can we determine which are the most effective actions that a manager can perform to maximize the engagement of participants?

3. Can we build different profiles of users based on their behavior during the challenge? 
We applied our model by performing a large set of experiments in the context of a real-life scenario, called YourExpo2015, which featured about 750,000 social activities over a period of 9 weeks, with 9 corresponding challenges launched. We developed the challenge progression in a mixed top-down and bottom-up approach: on one hand, in every challenge we had the freedom of deciding engagement policies; on the other hand, direct experience in one challenge suggested how to define or modify key elements in the next one. Observation of the challenges allow us to draw interesting conclusions on the effectiveness of the various actions that we performed (as managers) or induced (on the players, fans, and owners).

The paper is structured as follows. We start with the related work analysis; then we define a model of the actors, the actions, and their interplay. Then we briefly present our technological framework and we extensively discuss our experiments within the YourExpo2 015 challenge, and we show the effect of the various engagement policies. Finally, we discuss the results and conclude.

\section{Related Work}

Several works focused on analyzing and mining users behavior on social networks [21/4]8]. We are not aware of work specifically dedicated to the engagement is social network challenges; we therefore describe social engagement in other contexts. We use the gaming paradigm of a photo challenge for engaging users and increase awareness; this is in line with the gamification approach [5]Reward and reputation systems are at the core of gamified applications, which builds upon incentive-centered design. This is studied in persuasive technology [7], where games are seen as means to shape user behavior [12]14], or to instill desired values [1]. Factors that influence human behavior are fundamentally of two types, namely, incentive and cost [13|16]. The former increases and the latter decreases the motivation to complete an action. In this paper we experimented with purely external and immaterial incentives, i.e., visibility gain [15]. User motivations are very heterogeneous, and in particular they vary a lot depending on the scenario [20] and on incentives can be dynamically tuned [2] or combined [17].

Engagement has been often measured in the context of online applications measuring the actions performed by the users, such as page views, click-through rates and return rates [11]. These metrics have been used as basis for our measurement and mapped to the concepts provided by the different platforms.

Various works studied the incentives and conditions that favor participation in In the context of social networks, Irena at al. [3] study which are the most important factors for customer engagement in the domain of a Facebook brand page. Yogo et al. study incentives that stimulate activities in social networking services [22], while [19] examine the conditions that encourage users to participate more intensively in social networking. [6] studied the trade-off between the cost represented by the concern about personal data privacy and the incentive of sharing personal facts, records or content.

Similarly to what we do, Kumar et al. classify social network users into roles [10] and [18] study specific roles for the content tagging activity; however, our roles are specific for challenge scenarios. Finally, some studies on social media try to understand at which time the most social activity occurs [9]. 


\section{Modeling Social Network Challenges}

In the paper we focus on the specific case of content production challenges, i.e., any kind of online games where users are requested to submit original content (photos, videos, text or any other media). We start by describing a model of social network challenge, as shown in Fig. 1. the model includes the actors, the actions, their interplay in content production games, and external factors that influence engagement.

\subsection{Actors}

A social challenge requires the interplay of four kinds of actors.

- Manager. Sets the rules of the game, typically encoded in the game regulations, and then performs the activities which are prescribed by such rules. In addition, the organizer performs activities targeted to enhancing social participation, such as boosting visibility of the top players.

- Player. Autonomously decides to play the game, typically for obtaining visibility for themselves or for their content. Visibility is granted either by other members of the social network or by the organizers of the challenge (or both).

- Fan. Follows the evolution of the game and decides the outcome by voting on the content produced by the players.

- Owner. Initiates the challenge based on some business or marketing need. According to that, he assigns the practical execution of the challenge to the manager. Owners include popular social accounts (actors, celebrities, institutions, well known brands), or real world events that have a strong impact on the public (such as TV programs, commercial advertising, endorsement by government or large companies). Their high visibility supports the managers in boosting the social participation; their actions may or may not be under the control of the organizers, and thus the consequences may be hard to predict.

\subsection{Activities and Actions}

We identify four main kinds of activities which contribute to a social challenge, each embodied in several actions.

- Invite. The purpose of inviting is to make the challenge known on the social network and to convince potential players to participate. These actions may imply publishing of direct descriptions of the challenge, or evocative contents for the topic or focus of the challenge.

- Contribute. The purpose is to participate to the game on the social platform of choice. For content production games, this implies to submit original content (photos, videos or text) produced by the player, specifically devised for the challenge and prepared during the challenge period. Some challenges may allow the player to post content that was pre-existing to the challenge. This entails both content produced by other people (e.g., photos or videos found on the web) or content produced by the player himself for other reasons (typically in the past) and reused for the challenge. If the challenge is asking to enrich existing content, the contribution 


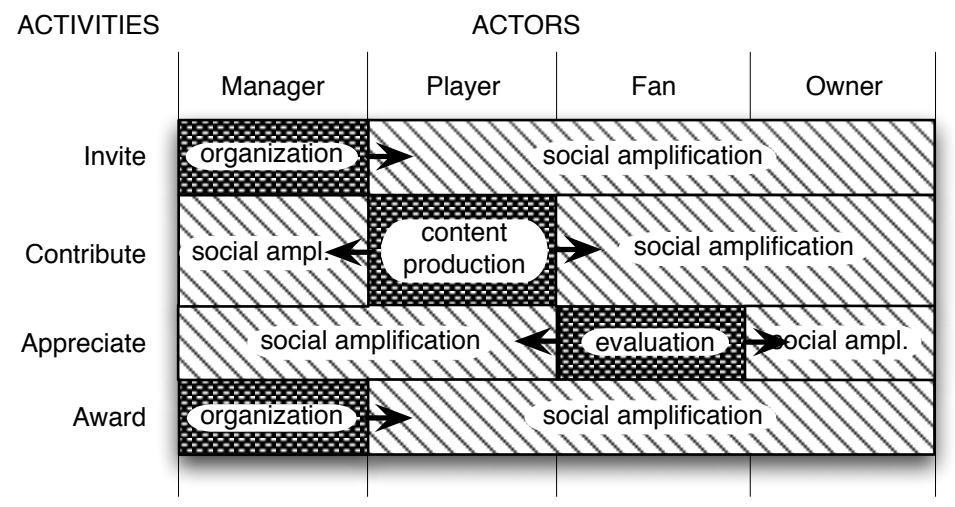

Fig. 1: Model of actors, activities and amplification effect in a social challenge.

may consist in expanding the content descriptions, through adding tags, location, text, people or other entities to the content; or by identifying similar items or listing the contents in some specific order or ranking.

- Appreciate. The purpose is to express and share appreciation for the activities or content of a player, or for the participant himself, for instance by liking the content, i.e., annotating the content with a "like" or "preference" tag (typical in social networks like Facebook, Instagram or Twitter), or by following the player, i.e., declaring interest in the person and following his activities.

Invite and award actions are typically performed by the manager; contribute is performed by players; while appreciate actions are performed by the fans, but can be tactically performed by organizers and players too, in order to enhance the social participation. Typically, owners do not engage directly into appreciation actions, because they must appear as neutral, and their size and visibility is incomparable to those of players.

The peculiar case of social-network based games enables an additional behaviour, which is to generate Social Amplification of the actions, through social sharing. Social amplification can be performed by any actor, upon the activity of any other. The effect is that any activity can be made more visible and appreciated by a wider audience, based on the visibility of the actor that performs the sharing. A crucial role in social amplification is played by the owner, whose sharing activity can dramatically boost visibility.

\subsection{Other Aspects that Influence Engagement}

Aspects such as the challenge's staging, timing and multiplatform execution should be also considered in organizing a challenge.

Staging A challenge can be a single-shot event, where players make their actions and fans vote within a short time interval. However, an important aspect of social challenges is to build loyalty, which occurs when the players and fans become acquainted 


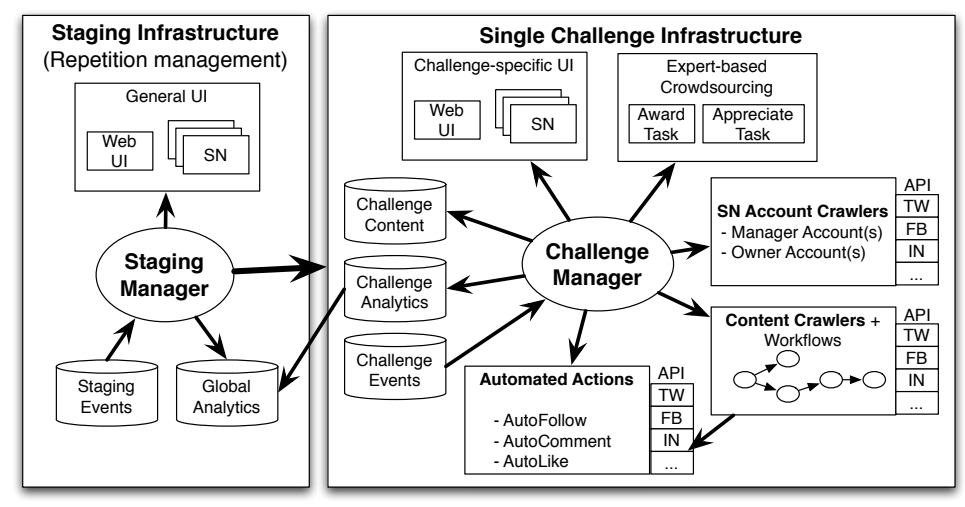

Fig. 2: Architecture of the system that manages and monitors the challenge.

with the game and repeat their actions many times. Loyalty can only occur when the game is staged, i.e., it is structured in a way that allows a player to anticipate the game progression and engage in a multi-action participation. Staging can be obtained by:

- Repeating the challenge periodically, with a winner for each period.

- Sub-structuring the challenge into phases, and giving to the player different task to perform at each stage.

Influence of daytime Social amplification of actions is extremely different depending on the time at which the action is performed and also of the particular day (e.g., workday/ weekend/ vacation). Time-dependent effects must be studied for each challenge, e.g. certain challenges may attract higher participation during night time or weekends.

Cross-Social Network Fertilization Cross-network fertilization is made possible because most actors participate in multiple social networks at the same time (e.g., they share content on Instagram and Twitter, and have friends on Facebook and collaborators on LinkedIn). Thus, it is possible to influence their behaviour on one social network through actions that occur on another one.

\section{Implementation}

We designed and implemented an architecture for analyzing and managing staged social challenges, shown in Fig. 2 The architecture is layered, with an external layer, called staging infrastructure, which generates several versions of the internal layer, called single challenge infrastructure. They both are associated with several resources dedicated to user interaction. Normally, these include a Web Interface which publishes the rules of the challenge, and several social network pages, describing the specific portion of the challenge which takes place on each social network. These resources may be present only at the stage level, if each stage is managed as an independent challenge. 
The staging manager holds information about the series of events which are associated with each stage, and in addition it manages global data analytics obtained as the summary of the various stages. The organization of the challenge core is performed by the challenge manager. It instantiates and manages several crawlers belonging to predefined classes, that are addressed to specific social networks and perform specific tasks, which are either content-specifications or account monitoring on each social networks; some of them are automatic actions generated in response to the task's output (e.g., following accounts or liking contents). Some of the content-specific tasks need to be organized through mini-workflows (e.g., the monitoring of likes given to a specific content must follow the post of that content.) Crawlers activities are timed so as to respect the constraints on API usage which are imposed by each social network; content is accessed through the given challenge hashtag, generated by the staging infrastructure and used as parameter in the calls to the social network APIs.

Data collected by crawlers are stored into stage-specific data analytics. Thanks to several sensing techniques over the challenge evolution, it is possible to understand and classify the behavior of players in terms of amount of activity, continuity of the actions in time, reactivity to solicitation from organizers, willingness to share challenge content and messages of the challenge, and extent of social amplification. Finalists and winners can be automatically determined by the system based upon such analytics.

\section{Experiments on YourExpo2015}

Expo 2015 The Universal Exhibition Expo 2015 was hosted in Milano; it received over 20 million visitors in its 1.1 million square meters of exhibition area. Over a six-month period, more than 140 participating countries run their pavillons around the theme of guaranteeing healthy, safe and sufficient food for everyone. Expo 2015 created a number of marketing campaigns on traditional and social channels. In such framework, we performed a set of experiments for increasing brand awareness of Expo 2015 before the event, through the development of a photo challenge, called YourExpo2015. The challenge has been independently managed by us, but has benefited of some interaction with the official social accounts of Expo 2015; their actions can be considered as owner activities according to our model. The objective of the experiment was two-fold: first, we wanted to verify the effectiveness of our model in a real world scenario; second, we wanted to respond to our research questions, and thus get insights on how the various actors behave, in order to get information useful for organizing future challenges.

\subsection{Purpose and Actions of the Challenge}

The game YourExpo2015 is based on the social production of photos on Instagram, in response to specific hashtags which are published every week by Expo 2015. The purpose of the challenge is twofold: to engage users for increasing the visibility of the Expo 2015 brand, and to collect relevant content associated to the Expo idea and purpose. The challenge proposes at every stage two hashtags, paired in a way that hints to a contrast (e.g., Fast/Slow, Art/Fun, Land/Sea, and so on). Although most posted photos show food, they can be on arbitrary subjects. The best photo for each 
of the two hashtags, separately selected, compose a postcard where the two photos and hashtags are shown together; as the main challenge reward, Expo 2015 used the postcards as header of its Facebook page. The challenge run for 9 weeks between Dec. 7, 2014 and February 21, 2015, with one pair of hashtag published every week.

Within the challenge we applied the model described in Section 3 , in particular, we devised two types of Invite actions:

- Announce: explicitly declares the start of the challenge, through posting of rules of engagement, deadlines, or aim of the game.

- Recall: sends our reminders and repetitive messages about the topics, the game rules, and the duration of the challenge.

We also covered Awards by mentioning players in three ways:

- Composite: a selection of four good photos posted until a given moment, with explicit mention of the social accounts of the respective authors.

- Finalists: the selection of the four best photos of the current week, with explicit mention of the authors. The selection is posted on two different social networks. These photos enter the voting for the winner of that week.

- Winners: the post advertising the winners of the week.

Fig. 3 shows a typical history of votes (likes) expressed upon few photos, from the initial posting of photos to the definition of the winner.

A few days after the start of the stage (Announce action), we repost the most voted photos in a Composite, that consists of four photos. Then, we select the Finalists and we publish them on Facebook; with a small delay we republish a Finalist post on Instagram too, so that the finalists (whose identity on Facebook is not known to us) come to know about the selection, and they start a second round of promotion through their Facebook friends, thereby performing cross-platform engagement. Eventually, the winners are selected (by counting its Facebook votes) and advertised through specific Winner posts.

\subsection{Structure and Rationale of the Experiments}

We run 9 different experiments, corresponding to the 9 weeks of the challenge, instantiated according to 4 different configurations, so that each experiment was repeated at least 2 times within each configuration. When setting up each experiment, we relied on the insight gathered during the previous runs in order to define significant variations of the configuration. The four configurations devised for our experiments were:

- Amplification by Owner (first configuration): each challenge is run on Instagram and starts with an announcement post. During the week additional recall posts are issued, to remind about the ongoing challenge theme. The social amplification action of the owner is scheduled on the recall post on the third day of the challenge.

- Amplification by Manager (second configuration): with respect to the first configuration, we added the composite posts. They were issued two times a day, so as to create competition and engagement around the selection. 


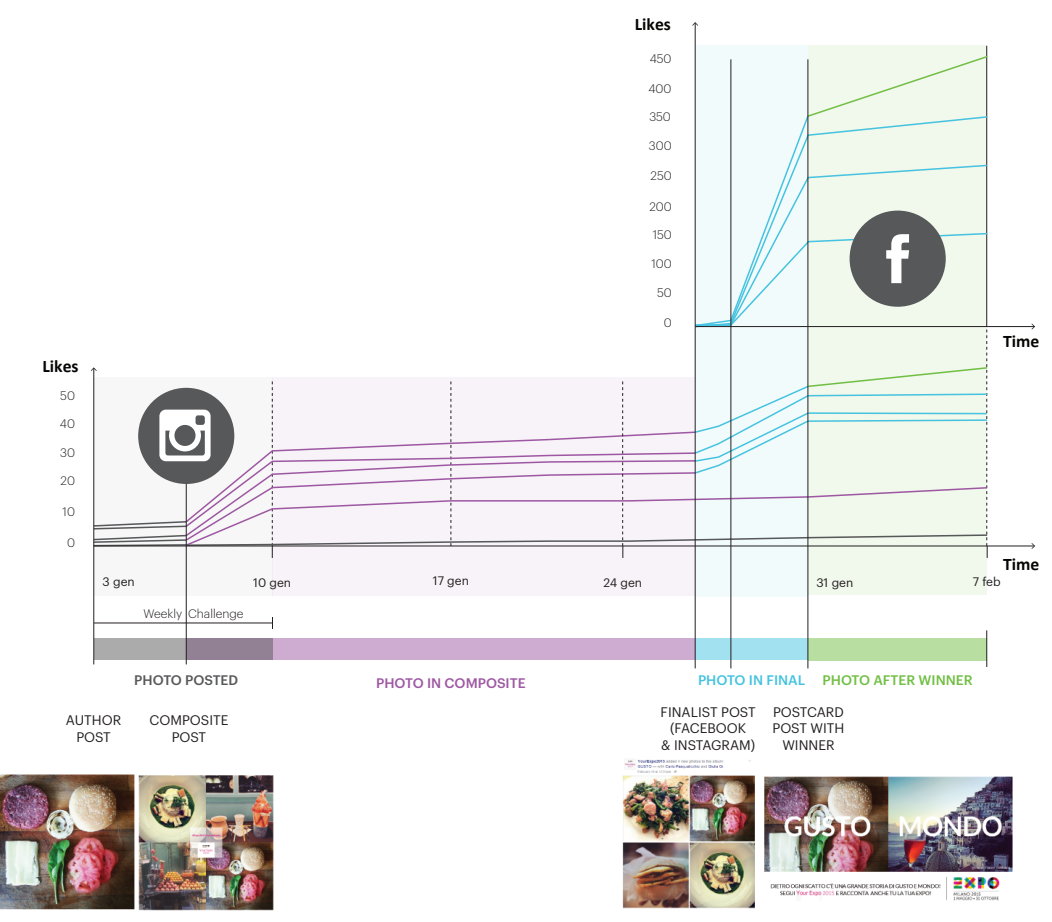

Fig. 3: Storyboard of a winning photo and monitoring of appreciation across social network.

- Cross-platform Amplification (third configuration): we expanded the action to multiple social network, namely including Facebook: every week we selected the four most liked photos as finalists on Facebook (and subsequently on Instagram), asking to vote for the best. Eventually, the winners are selected. In this configuration we also added an automatic like (by the account of the challenge manager) to every photo submitted to the challenge. Additionally, with respect to the second configuration, we increased the number of award actions, by adding the selection of finalists and winners. The actions of the owner were scheduled on the fourth, sixth and seventh days of challenge (Thursday, Saturday and Sunday) and consisted in a share of our announcement, recall and award posts.

- Amplification without Owner (fourth configuration): in the final configuration, we run the challenge without the help of the owner, i.e., with no social amplification by the owner. We also added an automatic action by the manager, i.e., the automatic follow of all the players in the current challenge.

The expected result of this structure of experiment is to obtain a coherent pattern of engagement, based on our actions. We consider the role of the owne asr particularly relevant. Therefore, our expectation is to have a pattern with significant peaks of engagement for every major social amplification performed by the owner. 


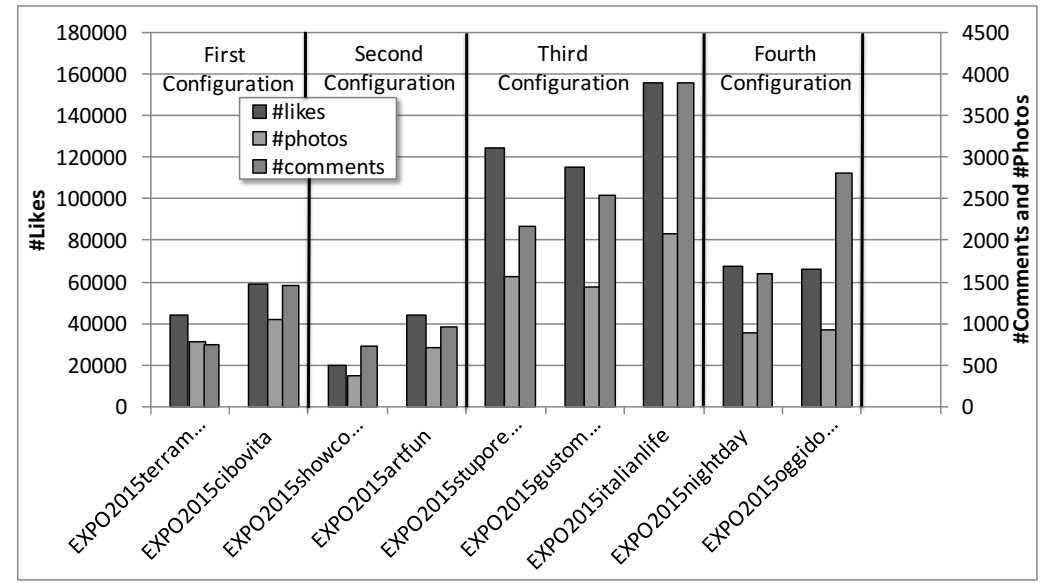

Fig. 4: Total number of likes, photos and comments for each experiment, grouped based on the different configurations.

\section{Experiment Results and Discussion}

\subsection{Players Participation}

The complete cycle of nine weeks of challenges generated more than 600,000 actions (post, like, comment) on Instagram and about 150,000 contacts on Facebook, with more than 3,000 followers on Instagram and more than 2,000 followers on Facebook.

Fig. 4 shows the distribution of likes, comments, and photo posts on Instagram, for each run of the challenge. The figure shows that in the third configuration, thanks to the actions we put in place, we obtained a very good level of engagement. Conversely, in the second configuration the engagement was rather low, but this was due to external conditions (Christmas and New Year's holiday weeks). The relative drop of engagement in the fourth configuration (where we removed the actions of the owner) confirms the prominence of the owner's role.

\subsection{Reactions to Actions of Organizers}

Besides monitoring the overall success of the challenge in terms of photos posted, liked and commented by players and fans, we also analyzed the response of the audience to specific individual solicitations.

We verified that the different types of posts by the challenge manager generated significantly different reactions of participants. For this test, we separated our actions in classes and performed the $t$-test on the classes, comparing the distribution of likes and comments of each group. The null hypothesis is that the different types of posts generate the same amount of interaction. We used the Welch's t-test, since the limited non-normality of our data and because the assumption of homogeneity of variances failed. We also applied the Holm-Bonferroni correction for multiple tests. We run our 


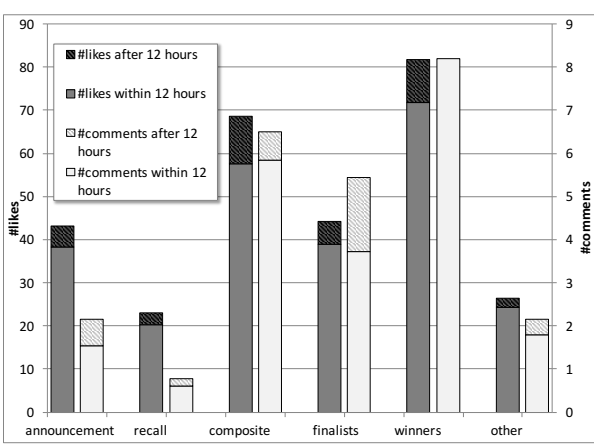

(a)

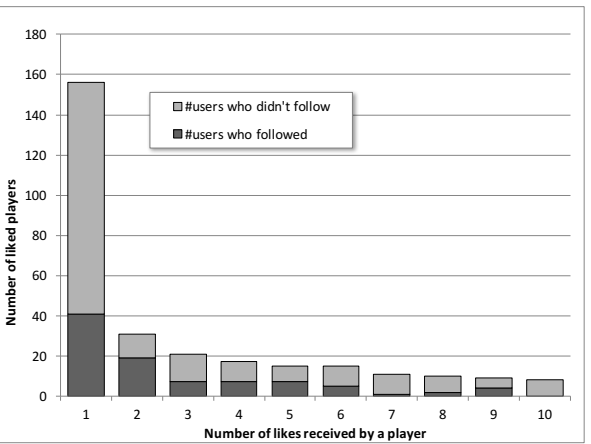

(b)

Fig. 5: Average number of likes and comments received on the different types of our posts on Instagram (a); and number of players who followed the official account of the challenge versus the number of likes received (b).

tests on two levels of granularity. Based on our model (see Section 3.2), we first contrasted invite actions (announcement and recall) with award actions (composite, finalists, and winners). Results of the test are acceptable, with $p$-value $<0.001$ both for likes and comments.

Subsequently, we tested more in detail how the reactions to the different actions differ from each other, by using the six classes defined above. Using the number of likes as observed feature, the $p$-value is acceptable for most pairs of classes ( 11 over a total of 15 pairs), confirming that the actions generated significantly different reactions. For what concerns comments instead, the t-test failed in most of the cases. Indeed, comments differently from likes, they are hardly triggered by external events, because people decide to comment on content when they are driven by very specific personal motivations.

Fig. 5(a) shows the number of likes and comments to our posts on Instagram, based on the type of post; the diagram shows that composites and winners got the maximum number of reactions. Most of reactions take place in the 12 hours immediately following the posts.

An interesting feature of our challenge architecture is the ability to automatically perform actions in response to the activities of players, that we monitor through crawlers. In particular, in the third configuration we generated automatic likes to every posts, and we inspected the player's response to such automatic likes in the form of a follow action to the official challenge account; in Fig. 55 (b) the reaction is plotted as a function of the total number of likes received by each user. It turns out that the first and especially the second like got the strongest reaction (respectively with around 25\% and 60\% of follow backs), while subsequent likes were less effective.

In the fourth configuration we also organized an automatic follow action to players, and we monitored the player's responses to our action; Fig. 6 shows such responses. In general, we can see that most reactions occur within the first two-three hours. In particular, as shown in Fig. 6(a), follow-back reactions are fast if the user are followed 


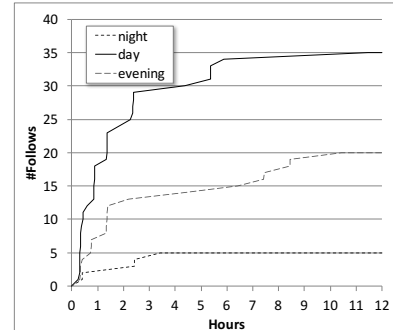

(a)

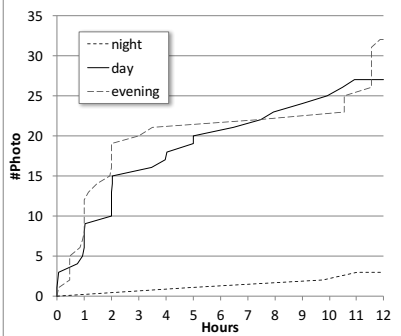

(b)

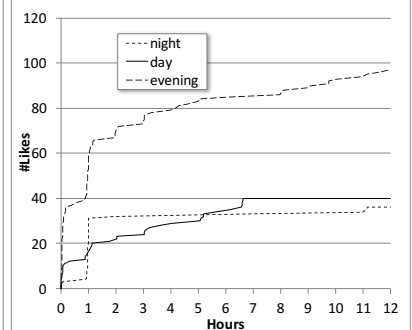

(c)

Fig. 6: Numbers new follows (a), new photos (b) and likes (c) as reactions to our automatic follow actions, for each time slot when our action was issued. The night time slot is between midnight and 9am. Day is between 9am and 5pm, while evening corresponds to the time between $5 \mathrm{pm}$ and midnight.

during the day, while they are very slow during the night. Fig. 6(b) shows the number of photos posted after our follow. This type of reaction is very fast both during the day and the evening. Finally Fig. 6a) shows the number of like reactions. In this case the reactions are fast in all the time slots of the day, as this action is less demanding.

In terms of volume, we noticed that the actions of players and participants are heavily influenced by the daytime, with the majority of activities taking place from 7 am to midnight, as expected, with peaks at lunch time and after dinner.

Fig. 7 shows the distribution of the reactions in the different time slots (night, from midnight to $9 \mathrm{am}$; day, from $9 \mathrm{am}$ to $5 \mathrm{pm}$; evening, from $5 \mathrm{pm}$ to midnight). During night hours the majority of reactions are of type like, with very limited amount of posts and follow actions. During the day and evening the share of new photos (and follow actions) increases significantly.

\subsection{Cluster Analysis of Players}

We applied cluster analysis to determine how users are involved in the challenge, either as players or as voters; to evaluate their behavior, we selected the number of likes and number of posted photos as dimensions. We first removed the outliers that were qualitatively detected as the account of the organizer (YourExpo2015) and all the users that liked only one image and never posted a photo (100k over 160k users), then we run the k-mean cluster algorithms, which produced the clusters, shown in Fig. 8 The number of clusters was determined using the Elbow Method, that consists in stopping the k-mean algorithm when adding a new cluster does not decrease the squared distance between each member of the cluster and its centroid. Clusters 7 and 1 group users who specialized as players and voters respectively; then, clusters 6 and 4 group users who were less active but still specialized. The other clusters include users which were active in both roles, with decreasing activity going from cluster 3 to clusters 2 and 5. Clusters 6 and 7 identify the most active players (158 users), while cluster 5 represents the least active users (57504 users). 


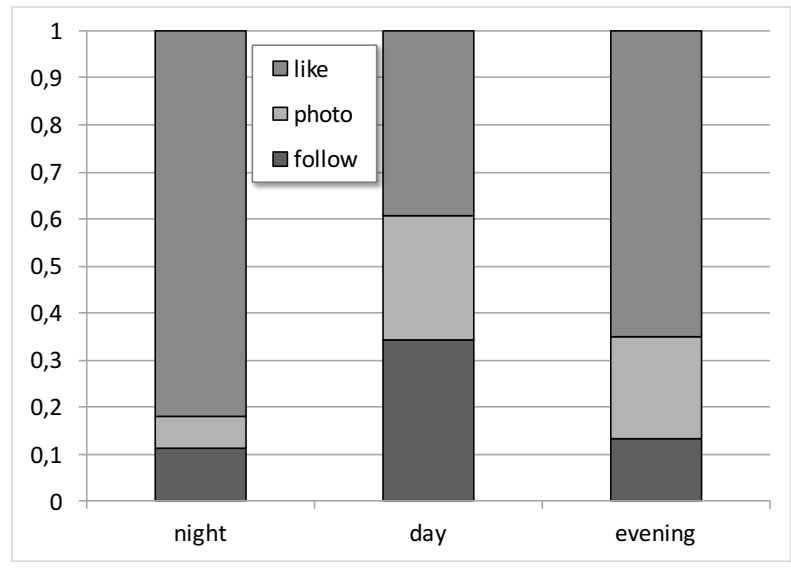

Fig. 7: Distribution of reactions to automatic follow actions upon posting of a photo, during three different daytime slots.

Clustering of users could be useful for increasing the success of a challenge, by addressing each type of message to a specific cluster of users. For instance, if the objective is to increase the collected content in a creation challenge, the organizer should leverage clusters 7 and 6; conversely, solicitation of votes or likes would should be addressed to users of cluster 1 .

\subsection{Discussion}

We close this section by summarizing the insights obtained with this experiment. It is clearly important to use each social network at its best: in our experience we used Instagram for posting and Facebook for final voting, while our attempts to propagate attention using Twitter were less successful. Indeed, our challenge had no significant/unexpected events, and thus it was not a suitable target for Twitter, that is notoriously very effective in spreading information about big events, such as terrorist attacks, but also comparatively minor events, such as TED conferences.

It is quite important to produce regular challenges, e.g., through periodic staging, so that many players may repeat their actions several times (e.g., several players of YourExpo2015 posted tens of photos); repetition and long duration also helps in growing a large and fidelized audience. It is also important to be fully aware of temporal factors, e.g., daytime or festivities, in order to properly plan automatic and owner actions that may engage new players. Temporal factors must be considered for any kind of action, including the automatic ones which are useless/negative if posted when people don't react.

Mere announcements of challenges and call to actions fail to engage people, but active management by managers pays. In particular, mentioning players is viral, especially when several of them are mentioned together, because this action stirs interactions among them, even if they do not know each other in advance. Although players want to win, the development of social relationship is perhaps an even stronger driver, therefore 


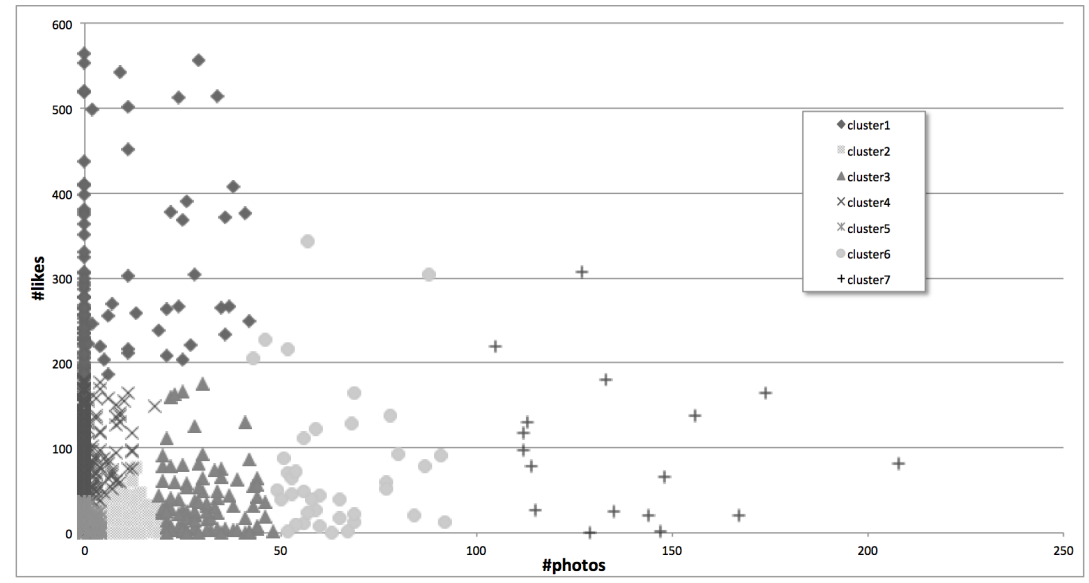

Fig. 8: Clusters of users.

organizers should spend lots of efforts in creating mutual engagement. In our experiments, composite mentioning of several players created a sub-network of participants who positively interacted, both by contributing content and by mobilizing voters. This constitutes a notable difference with respect to traditional crowdsourcing platforms, where instead workers are not engaged by mutual actions or interactions.

Visibility provided by owner actions is fundamental to boost the challenge and to keep it alive; assuming that owner actions are scarce / expensive resources, they must be programmed in a way that provides maximum effect upon the players.

\section{Conclusions}

In this paper, we defined a model of the actors and activities involved in a social network challenge; we demonstrated that social platforms are effective in engaging players in games for content generation or enrichment, and we illustrated how the different choices of game organizers impact on the quality and quantity of player's engagement. Referring to our initial research questions, we can claim that:

1. We modeled different roles, which indeed perform different activities in the challenge, as reported also in our experiments;

2. We studied the different types of actions performed, and monitored which ones are most effective action types with respect to the engagement;

3. We cluste red the users in profile "groups" based on their behavior.

Of course, the experimental results reported in this paper are relative to our context, but many of them (e.g., those on individual engagement and on social reaction rules and their timings) have general validity. The main objective of social challenges is to boost social interest (on events, brands, etc.), but as a side effect valuable content is produced or improved; thus, our model applies to an interesting class of games with a purpose, 
where users produce results by being moved by non-monetary rewards. We are currently planning an application of the method to brand promotion with large companies, e.g. in fashion.

\section{References}

1. P. Barr, J. Noble, and R. Biddle. Video game values: Human-computer interaction and games. Interacting with Computers, 19(2):180-195, 2007.

2. R. Cheng and J. Vassileva. Design and evaluation of an adaptive incentive mechanism for sustained educational online communities. User Model. User-Adapt. Interact., 16(3-4):321348, 2006.

3. I. P. Cvijikj and F. Michahelles. Online engagement factors on facebook brand pages. Social Network Analysis and Mining, 3(4):843-861, 2013.

4. A. Das, S. Gollapudi, and K. Munagala. Modeling opinion dynamics in social networks. In 7th ACM Conference on Web Search and Data Mining, WSDM 2014, pages 403-412, 2014.

5. S. Deterding, M. Sicart, L. Nacke, K. O'Hara, and D. Dixon. Gamification. using gamedesign elements in non-gaming contexts. In International Conference on Human Factors in Computing Systems, CHI 2011, Vancouver, BC, Canada, May 7-12, 2011, pages 2425-2428, 2011.

6. C. Dwyer. Digital relationships in the "myspace" generation: Results from a qualitative study. In 40th Hawaii International International Conference on Systems Science (HICSS40 2007), 2007, USA, page 19, 2007.

7. B. J. Fogg. Persuasive technology: Using computers to change what we think and do. Ubiquity, 2002(December), Dec. 2002.

8. S. Gao, J. Ma, and Z. Chen. Modeling and predicting retweeting dynamics on microblogging platforms. In 8th ACM Conference on Web Search and Data Mining, WSDM 2015, pages $107-116,2015$.

9. S. Golder, D. Wilkinson, and B. Huberman. Rhythms of social interaction: Messaging within a massive online network. In C. Steinfield, B. Pentland, M. Ackerman, and N. Contractor, editors, Communities and Technologies 2007, pages 41-66. Springer London, 2007.

10. R. Kumar, J. Novak, and A. Tomkins. Structure and evolution of online social networks. In 12th ACM SIGKDD International Conference on Knowledge Discovery and Data Mining, Philadelphia, PA, pages 611-617, 2006.

11. M. Lalmas, H. O'Brien, and E. Yom-Tov. Measuring User Engagement. Synthesis Lectures on Information Concepts, Retrieval, and Services. Morgan \& Claypool Publishers, 2014.

12. D. Lockton, D. Harrison, and N. A. Stanton. The design with intent method: A design tool for influencing user behaviour. Applied Ergonomics, 41(3):382-392, 2010.

13. P. Milgrom and J. Roberts. Economics, organization and management, 1992.

14. S. Niebuhr and D. Kerkow. Captivating patterns - A first validation. In Second International Conference on Persuasive Technology, PERSUASIVE 2007, Palo Alto, CA, USA, 2007, pages 48-54, 2007.

15. A. M. Rashid, K. S. Ling, R. D. Tassone, P. Resnick, R. E. Kraut, and J. Riedl. Motivating participation by displaying the value of contribution. In Conference on Human Factors in Computing Systems, CHI 2006, Montréal, Canada, April 22-27, pages 955-958, 2006.

16. K. Sato, R. Hashimoto, M. Yoshino, R. Shinkuma, and T. Takahashi. Incentive mechanism considering variety of user cost in $\mathrm{p} 2 \mathrm{p}$ content sharing. In IEEE Global Telecommunications Conference (GLOBECOM) 2008, pages 1-5, Nov 2008.

17. O. Scekic, H. L. Truong, and S. Dustdar. Incentives and rewarding in social computing. Commun. ACM, 56(6):72-82, 2013. 
18. J. Thom-Santelli, M. J. Muller, and D. R. Millen. Social tagging roles: publishers, evangelists, leaders. In Conference on Human Factors in Computing Systems, CHI 2008, Florence, Italy, pages 1041-1044, 2008.

19. F. Toriumi, K. Ishida, and K. Ishii. Encouragement methods for small social network services. In 2008 IEEE / WIC / ACM International Conference on Web Intelligence, WI 2008, Sydney, pages 84-90, 2008.

20. M. M. Wasko and S. Faraj. Why should I share? examining social capital and knowledge contribution in electronic networks of practice. MIS Quarterly, 29(1):35-57, 2005.

21. S. Wu, A. D. Sarma, A. Fabrikant, S. Lattanzi, and A. Tomkins. Arrival and departure dynamics in social networks. In 6th ACM Conference on Web Search and Data Mining, WSDM 2013, pages 233-242, 2013.

22. K. Yogo, R. Shinkuma, T. Konishi, S. Itaya, S. Doi, K. Yamada, and T. Takahashi. Incentiverewarding mechanism to stimulate activities in social networking services. Int. Journal of Network Management, 22(1):1-11, 2012. 